\title{
El territorio de las nuevas economías metropolitanas
}

\begin{abstract}
The recent economic changes in metropolitan regions have given rise to significant shifts in the locational strategies of firms, the spatial organisation of different activities, functions and jobs, with direct consequences for urban form. A diagnosis of the new economic geography of world cities and metropolitan regions is a key factor in shaping new economic promotion policies and urban planning. This paper offers a critical review of diverse concepts and theories relating to the recent territorial evolution of metropolitan economies, it analyses the recent trends in spatial organisation of activities, and it emphasises the strategic value of local responses in the construction of competitive and innovative cities.
\end{abstract}

Key words: metropolitan economy, urban form, location of activities, territorial models of innovation.

\section{Resumen}

Las transformaciones económicas recientes ocurridas en las regiones metropolitanas suponen importantes cambios en las pautas de localización de las empresas, el dinamismo y la organización espacial de las diferentes actividades, empleos y funciones, con evidentes efectos sobre la forma urbana. Un diagnóstico sobre la nueva geografía económica de las ciudades mundiales y sus regiones metropolitanas es un factor clave para orientar las nuevas políticas de promoción económica y planeamiento urbanístico. El artículo propone una revisión crítica de diferentes conceptos y teorías sobre la actual relación entre economías y territorios metropolitanos, analiza las tendencias recientes en la organización espacial de las actividades y destaca la importancia de las respuestas locales en la construcción de ciudades competitivas e innovadoras.

Palabras clave: economía metropolitana, forma urbana, localización de actividades, modelos territoriales de innovación. 


\section{Introducción}

$\mathrm{L}$ as grandes aglomeraciones urbanas constituyen un observatorio privilegiado para describir, analizar e interpretar buena parte de las transformaciones asociadas a la nueva fase de desarrollo capitalista. La evolución reciente, tanto de su estructura y morfología internas como de sus límites externos y sus relaciones con el entorno, afectan el funcionamiento de sus empresas, el volumen y calidad del empleo, así como la vida de sus ciudadanos, exigiendo nuevas formas de pensar y actuar sobre ellas.

En el plano de la actividad económica, se modifican las estrategias competitivas de las empresas para adaptarse al nuevo contexto tecnológico, a modelos de organización más flexibles y segmentados, así como a unos mercados en donde su creciente apertura y ampliación genera nuevas oportunidades, pero también hace surgir nuevos competidores y demandas de innovación que no todos están en condiciones de afrontar. Se transforman, en paralelo, unos mercados de trabajo en los que las mayores exigencias de formación y la aparición de nuevos yacimientos de empleo van, a menudo, de la mano con la difusión de formas de trabajo precarias e inestables. Se diversifican, por último, las trayectorias espaciales de las diferentes actividades, funciones, empresas y empleos, lo que refuerza la especialización y jerarquización de los territorios.

Transformaciones que se asocian de forma directa a nuevas formas urbanas, que han dado origen a una amplia proliferación de neologismos y metáforas en los últimos años, que se superponen a los de metrópolis, conurbación o megalópolis, de más larga tradición, y ponen de manifiesto las dificultades del lenguaje para proporcionar instrumentos capaces de describir adecuadamente la nueva urbe (Amendola, 2000, p. 35). En unos casos, hacen referencia a su contenido funcional y al protagonismo de algunas ciudades en la era del capitalismo global (ciudad postindustrial, global, mundial...), mientras en otros destacan la relación

Profesor de investigación, Instituto de Economía y Geografía CSIC, Madrid, España. E-mail: rmendez@ieg. csic.es. Enviado el 27 de marzo de 2007, aprobado el 13 de junio de 2007. entre nuevo marco tecnológico y estructura urbana (ciudad postfordista, informacional, digital, telépolis, ciberciudad, softcity...), el reforzamiento de los contrastes internos (ciudad collage, ciudad fractal, ciudad dual...), o suponen la integración de algunas en el marco de la llamada sociedad del conocimiento (ciudad inteligente, creativa...). Están también aquellos términos que aluden a un cambio de escala (megaciudad, hiperciudad, ciudad región...), o a su expansión más allá de unos límites bien definidos (ciudad dispersa, difusa, metrópolis policéntrica, metápolis, postsuburbia...). Síntesis de esos cambios y reflejo, a la vez, de esa inflación nominalista tan afín a determinadas visiones posmodernas, puede ser la postmetrópolis de Soja (2000), que es definida por este autor como flexcity, cosmopolis, exópolis, polarcity, carceral city y simcity al mismo tiempo. Algunos valiosos intentos recientes de poner orden en esta confusa maraña de denominaciones (Gaja, 2004; García Palomares, 2007) encuentran así serias dificultades para lograrlo.

Si bien es cierto que esto puede entenderse como manifestación del periodo de cambio intenso vivido en las últimas décadas, en bastantes ocasiones se trata de términos sugerentes, pero no tanto de conceptos demasiado precisos en la descripción de sus características o en las claves interpretativas de los procesos que subyacen a su construcción, al no responder a una interpretación teórica sobre las transformaciones urbanas estructurales del periodo actual, más allá de algunas de sus manifestaciones concretas. Como señala Ramírez (2006, p. 63), "subsiste una falta de claridad en los conceptos que usamos para definir el proceso urbano y, en ocasiones, los usamos automáticamente sin llegar realmente a contextualizarlo y caracterizarlo". De este modo, es frecuente la utilización de diversas denominaciones para aludir al mismo tipo de fenómeno, mientras en otras ocasiones un mismo término se utiliza con significados diversos según autores. También hay un evidente sesgo en la consideración de las ciudades estadounidenses -con Los Ángeles como paradigma- o de algunos ejemplos estudiados en regiones desarrolladas del mundo como modelo universal, que a menudo se pretende trasladar a territorios muy distintos, lo que conlleva evidentes excesos. Finalmente, parece existir cierta dicotomía entre la bibliogra- 
fía que se centra en el análisis de los procesos de base económica, tecnológica o sociocultural que transforman las metrópolis (Sassen, Taylor, Soja, Castells, Amendola...) y la que se preocupa, sobre todo, de las nuevas formas urbanas resultantes (Ascher, Garreau, Indovina, Dematteis, Nel.lo...), con escasa integración de ambas perspectivas.

Con ese marco de referencia, el presente texto se plantea como objetivo esbozar una interpretación sintética, de carácter teórico, sobre algunas dimensiones del cambio económico metropolitano, así como su reflejo sobre los límites externos, la estructura interna, el dinamismo y las nuevas formas de desigualdad presentes en las aglomeraciones urbanas. Propone, por tanto, que existen lógicas espaciales diferenciadas que subyacen a la apariencia, a menudo caótica, de esa ciudad sin confines (Nel. lo, 1998), fragmentada, dinámica y desigual, pero en donde las pautas de localización y de movilidad responden a estrategias bien definidas.

En concreto, esta propuesta interpretativa considera la diversidad de tendencias actuales a partir de la combinación de dos tipos de factores básicos. Están, por una parte, los procesos estructurales que responden a la nueva organización de la actividad productiva y el cambio en las estrategias competitivas urbanas, que reorientan las trayectorias económico-laborales y se traducen también en la dinamización de ciertos sectores de la aglomeración frente al declive de otros, al tiempo que se intensifican los procesos de difusión espacial y deslocalización en beneficio de territorios cada vez más alejados. Pero, junto a éstos, también es necesario abordar la diversidad de respuestas locales a partir de las características y formas de organización de los actores e instituciones locales, así como su mayor o menor capacidad para llevar adelante estrategias innovadoras en el ámbito de la promoción y la ordenación, a partir de unas herencias aún visibles en las trayectorias actuales. Aunque se trata de planteamientos con pretensiones generalizadoras, es indudable que bastantes afirmaciones están sesgadas por la experiencia personal y profesional del autor, por lo que pretenden deducir tendencias aplicables a las metrópolis del Sur de Europa y plantear el debate sobre su posible adecuación para comprender situaciones observables en otras regiones.

\section{Economías metropolitanas en el capitalis- mo global: algunas claves interpretativas.}

El aumento de la complejidad, asociado a transformaciones profundas y rápidas, alcanza su máxima expresión en las grandes metrópolis, cuyas economías y territorios han experimentado con intensidad los cambios estructurales que marcaron la transición hacia nuevas formas de organización del sistema. Por una parte, "ejercen funciones de elevada complejidad: proporcionar economías de aglomeración y proximidad, estimular la creatividad y la innovación, facilitar la accesibilidad e interacción social, integrarse en red con el mundo exterior y alcanzar un máximo bienestar colectivo", desempeñando un papel estratégico en el mundo globalizado, "ya que son el espacio en el que se localizan las funciones, actividades y servicios que estructuran el sistema económico internacional" (Cuadrado Roura y Fernández Güell, 2005, p. 67). Pero, al mismo tiempo, se enfrentan a un incremento de los costes económicos y sociales asociados a la congestión, la creciente competencia interurbana y la frecuente dificultad de las estructuras del gobierno local para gestionar con eficacia problemas que, a menudo, se escapan de su competencia. Se configuran así como "nuevos espacios de desarrollo, innovación y conflicto" (De Mattos et al., 2005, p. 13) en los que la evolución de sus actividades económicas constituye uno de los componentes constitutivos más relevantes para comprender esas contradicciones.

Tres son los rasgos de esa evolución que pueden considerarse de especial relevancia: la pervivencia de tendencias concentradoras que resisten la deslocalización selectiva de ciertas actividades e incorporan crecientes desigualdades cualitativas asociadas a la división espacial del trabajo; el reforzamiento de las economías metropolitanas en el mundo de las redes y la net-economía; por último, la transformación de la base económica metropolitana a favor de una creciente integración entre la industria y una parte de las actividades de servicios, que puede traducirse en trayectorias laborales diversas según el rango de las funciones que cada metrópoli es capaz de detentar en sistemas urbanos sometidos a una creciente competencia.

El primer aspecto a destacar es la inexistencia de una correlación directa entre los procesos de 
centralización/descentralización productiva con los de concentración/desconcentración espacial de las actividades, lo que no significa negar la existencia de relaciones entre las estrategias de acumulación y las de carácter territorial, sino cuestionar el modo concreto en que se materializan. Por ello, frente a las tesis de la contraurbanización y del declive metropolitano, que hace dos décadas pretendieron certificar el final del proceso de concentración espacial (Champion, Cheshire y Hay...) asociada a la supuesta postindustrialización y a la producción flexible, hoy resulta evidente el dinamismo económico - al menos en términos comparativos- que experimentan la mayor parte de las grandes aglomeraciones urbanas de nuestro entorno, reflejo de su capacidad para producir, distribuir, innovar y gestionar.

Perviven así fuertes ventajas competitivas para las metrópolis, tanto por el volumen de externalidades asociado al tamaño de sus mercados (de consumo, trabajo y capital), como por la densidad y calidad de sus infraestructuras físicas (desde vías de transporte de gran capacidad y aeropuertos internacionales, a plataformas logísticas, telepuertos, etc.) y, sobre todo, por toda una serie de recursos específicos hoy valorados como estratégicos. Éstos incluyen un capital humano cualificado, la presencia de organizaciones públicas y privadas generadoras o difusoras de conocimiento, así como un marco institucional generalmente favorable -al menos en términos comparativos con otros territorios- al intercambio de información, la apertura externa y la conformación de clusters empresariales y redes sociales potenciadoras de la innovación, con la aparición de rendimientos crecientes de escala (Fujita, Krugman y Venables, 2000; OCDE, 2006a). Por otra parte, la fuerte concentración en las metrópolis de las denominadas clases creativas (Florida, 2002; Scott, 2006) y de las actividades de servicios intensivas en conocimiento (OCDE, 2006b), no hace sino reforzar esa hegemonía en términos cualitativos, que ahora se suma a una polarización de actividades en términos cuantitativos que está lejos de haber desaparecido.

Al mismo tiempo, en el marco de una economía de redes, progresivamente interconectada y abierta, las metrópolis tienden a reforzar su tradicional funcionalidad como centros de poder y nodos de interconexión de los flujos -materiales e inmateriales- que vinculan a empresas y territorios. Esa constatación obliga a considerar, junto al espacio de los lugares en que se ubican materialmente las empresas y los empleos, un espacio de flujos que, como señaló Castells (1997, p. 411), "se está convirtiendo en la manifestación espacial dominante del poder y la funcionalidad en nuestras ciudades".

El origen de esa posición dominante de las metrópolis en el mundo de las redes es ya antiguo, pero su importancia resulta mucho más evidente ahora que la compresión espacio-temporal (Harvey, 1990), el aumento de los movimientos de capital transnacionales y la expansión de la nueva economía favorecen el reforzamiento de islas metropolitanas emergentes, interconectadas y jerarquizadas en red, depositarias de aquellas funciones estratégicas que controlan el sistema y generan mayor valor añadido, en contraste con amplios espacios sumergidos, que padecen los efectos perversos de esta globalización excluyente (Veltz, 1999).

De este modo, pese a la enorme complejidad de las múltiples redes que se superponen hoy en el territorio -lo que ha permitido el uso de analogías, a veces excesivas, procedentes de las ciencias físico-naturales, como aquellas que las describen en términos de geometría fractal o de campo rizomático-sus nodos centrales siguen identificados con algunas de las principales metrópolis existentes en nuestros países y todas compiten por generar las condiciones capaces de reforzar esa posición. Tal como afirma De Santiago (2006, p. 153), "en la nueva geografía sujeta a la inestabilidad de la competencia internacional, son más importantes las relaciones que las localizaciones, las posiciones en las redes productivas consolidadas que las unidades productivas, la capacidad decisional y de atracción de flujos que los asentamientos productivos". Las fuertes inversiones públicas y privadas de los últimos años para construir la infraestructura material que da soporte a los diferentes tipos de flujos -desde vías de transporte de gran capacidad a redes de banda ancha o plataformas logísticassupone un reflejo de la importancia que muchos actores locales suelen conceder a esa conexión, a veces en detrimento del esfuerzo destinado a 
enfrentar otros dilemas metropolitanos como el de la exclusión.

Una tercera característica común a todas las aglomeraciones metropolitanas es la evolución de su base económica en la dirección de una creciente terciarización, que reduce la participación relativa de la industria en el valor añadido total y su volumen de empleo neto, frente al constante incremento registrado por los servicios, que suele compensar con creces las pérdidas en el sector manufacturero.

La tesis de la metrópoli postindustrial señaló, hace ya tiempo, una tendencia que justificó por las deseconomías derivadas de la saturación de sus infraestructuras, la escasez y encarecimiento del suelo, la mayor presión fiscal o las crecientes restricciones urbanísticas y ambientales, junto a las expectativas de beneficio generadas por la recalificación del suelo hacia usos más intensivos, sobre todo cuando el planeamiento se limita a seguir los dictados del mercado, así como la atracción de nuevas áreas con menores costes o un entorno productivo más adecuado a las actuales necesidades de las empresa. Tal interpretación fue revisada hace ya dos décadas por las tesis neoindustriales, que vincularon una parte significativa del desplazamiento hacia los servicios a la nueva organización de la propia actividad productiva, con un aumento en la importancia de las tareas previas y posteriores a la fabricación, generadoras de valor añadido, que tienden a externalizarse y ser realizadas por empresas especializadas que, en términos estadísticos cambian de sector, pese a permanecer funcionalmente integradas (Caravaca y Méndez, 2003; Caravaca, 2006).

No obstante, el desarrollo de esos servicios a la producción, así como del llamado terciario direccional, continúa vinculado en gran parte a la existencia de una sólida base productiva, que actúa como soporte y factor de impulso. De lo contrario, las grandes ciudades pueden evolucionar hacia una pérdida de la complejidad funcional y la mezcla social que siempre se asoció con su propia vitalidad, para convertirse en centros casi exclusivos de distribución y consumo (Daniels y Bryson, 2002), lo que se traduce en trayectorias socioeconómicas muy diferentes bajo la simple referencia a la terciarización. En resumen, afirmar la primacía en las metrópolis de una economía de servicios resulta una evidencia banal, pues si en unos casos puede representar la evolución hacia servicios avanzados intensivos en conocimiento, actividades financieras o una mejora de los servicios públicos, en otros se traduce en el aumento de todo tipo de servicios personales y actividades comerciales de muy baja productividad, integrados a veces en el ámbito de la economía informal (Pradilla y Márquez, 2004).

La consideración de tales procesos desde una perspectiva funcional permite reinterpretar la actual tendencia evolutiva de muchas metrópolis hacia la construcción de economías servindustriales, en la que las tradicionales fronteras intersectoriales se diluyen en aras de una creciente integración, proceso ya muy avanzado en las grandes ciudades de las regiones más urbanizadas y prósperas, y que parece difundirse de forma jerárquica hacia otras de segundo nivel, si bien escasean los estudios realizados desde esta perspectiva como para llegar a afirmaciones contundentes en este sentido.

Con relación a las empresas industriales, esto conlleva una progresiva intensificación de la actividad en los establecimientos que mantienen en las metrópolis, aumentando el valor añadido que generan al tiempo que reducen el empleo directo, con un fuerte incremento de productividad. Al mismo tiempo, se refuerza la especialización metropolitana en sectores manufactureros intensivos en capital y tecnología, en detrimento de aquellos otros intensivos en recursos naturales, suelo o trabajo. Pero lo más relevante es la concentración que aquí registran las empresas que muestran mayor capacidad de innovación (en sus procesos, productos, gestión o acceso al mercado), cualquiera que sea su rama de actividad, así como de aquellas tareas previas o posteriores a la fabricación que constituyen el llamado terciario industrial, cuyo mejor exponente-aunque no único-son las sedes empresariales y oficinas industriales que en bastantes áreas sustituyen la anterior presencia de fábricas y talleres (Méndez, 2006).

En torno a ese conjunto de actividades productivas, crece también la presencia de sedes pertenecientes a empresas interesadas en las 
Figura 1. Actividades y funciones dinámicas en las economías servindustriales metropolitanas: efectos sobre el empleo

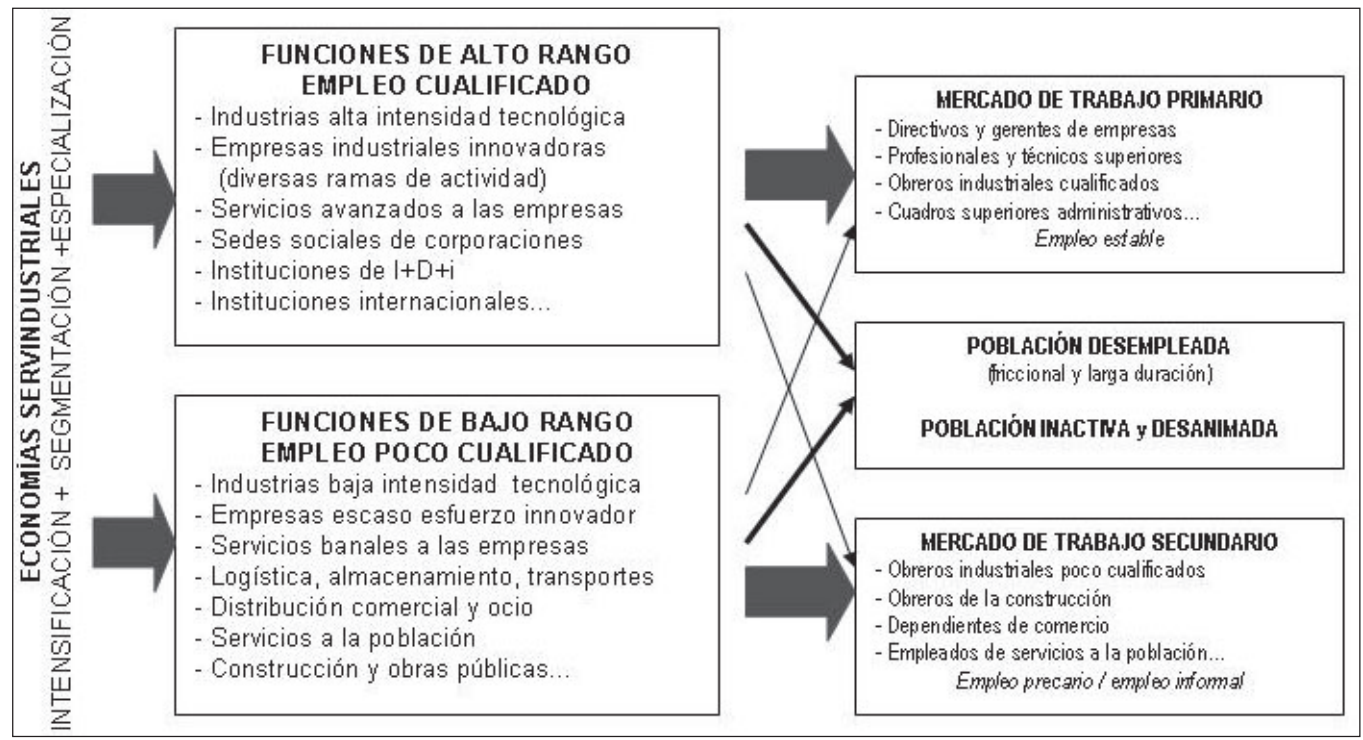

Fuente. Elaboración propia.

externalidades asociadas a la centralidad, junto a un amplio y creciente conjunto de servicios empresariales de alto nivel, externos a la industria, a los que se suman otros componentes del sistema local de innovación como pueden ser los centros de $\mathrm{I}+\mathrm{D}+\mathrm{i}$, las universidades, o aquellas instituciones y empresas que facilitan la conexión a las redes globales, desde las financieras a las vinculadas con las telecomunicaciones o los medios de comunicación y la industria cultural. Todo ello supone un conjunto de límites difusos, pero constituido por actividades de elevado rango funcional, que son también las de mayor resistencia a la deslocalización, tal como refleja la figura 1 .

Pero la consolidación de economías servindustriales puede asociarse también a la expansión de otro tipo de actividades y funciones de mucho menor rango, valor añadido o exigencias de cualificación profesional, aunque necesarias para el desarrollo de esta forma de acumulación. Son las relacionadas con la logística y el transporte de mercancías y personas, la distribución comercial mayorista y minorista, los servicios a la población y diversos servicios banales a las empresas (limpieza, mantenimiento, seguridad...), o el conjunto de actividades inmobiliarias y de construcción.
Aunque las formas de regulación imperantes en cada sociedad establecen diferencias a la hora de traducir estas tendencias económico-funcionales en el sistema de relaciones laborales, el resultado es una evolución de los empleos metropolitanos suficientemente compleja como para quedar mal reflejada en esquemas simplistas como los de la dualización (Mollenkopf y Castells, 1992; De Mattos, 2002), aunque eso no impide confirmar la presencia de trayectorias contrastadas -entre metrópolis y dentro de las mismas- en el marco de mercados de trabajo fuertemente segmentados.

En resumen, más allá de debates nominalistas que siguen a menudo los dictados de la moda, resulta imprescindible la revisión crítica de algunos conceptos de uso hoy frecuente, frente a la constatación de tendencias bastante más complejas y, a menudo, contradictorias. Algo similar puede afirmarse en relación a las nuevas formas metropolitanas, aspecto en el que las páginas que siguen centran su atención.

\section{La nueva organización espacial de las eco- nomías metropolitanas}

La forma urbana heredada por la ciudad mediterránea, que frente al modelo de ciudad 
imperante en otras regiones se caracterizó tradicionalmente por la existencia de un acusado gradiente densimétrico con máximos valores en las áreas centrales, tanto en términos de población como de actividad económica y empleo, junto a la identificación de unos límites externos bastante netos frente al entorno rural, se desdibuja hoy con rapidez. Si hace ya más de medio siglo la aparición del fenómeno metropolitano certificó el desbordamiento suburbano de las grandes ciudades, las transformaciones actuales suponen un nuevo salto -cuantitativo y cualitativo- que afecta de nuevo su fisonomía externa y organización interna, incluyendo la reorganización del mapa empresarial y en la distribución de los diversos tipos de ocupaciones.

Factor esencial de esa evolución es la transformación de las relaciones espacio-tiempo, generadora de una ampliación del campo de externalidad metropolitano (Dematteis, 1998), asociado al aumento de velocidad en los transportes y la instantaneidad en las telecomunicaciones. Tres son los rasgos de esta nueva geografía económica que con más frecuencia se repiten en la bibliografía internacional: la expansión del fenómeno metropolitano hasta alcanzar, en ocasiones, una dimensión regional; un cambio en su estructura interna que sustituye de forma progresiva los esquemas monocéntricos caracterizados por un acusado gradiente centro-periferia, por otros de carácter multipolar; y una ruptura en la continuidad como elemento definidor del espacio metropolitano, al considerar la existencia de áreas dispersas y a gran distancia del núcleo metropolitano, pero fuertemente interconectadas con él. El resultado de todo ello es un creciente protagonismo de las periferias metropolitanas como espacios de especial dinamismo y complejidad, sometidos también a fuertes tensiones derivadas de la competencia entre usos y la multiplicidad de actores e intereses que en ellos se confrontan.

En primer lugar, los límites urbanos se extienden en un movimiento que alcanza en ocasiones una dimensión regional, ante el que la pervivencia de unos límites administrativos estables se convierte en un elemento disfuncional. El concepto de región metropolitana, que en el ámbito internacional (UN-Habitat, OCDE, ESPON...) cuenta con diferentes definiciones y criterios para su delimitación, supone y exige un cambio de escala, al tratarse de un nuevo tipo de entidad, de carácter esencialmente funcional, que integra múltiples ciudades y una extensa área de influencia, de baja densidad y alta movilidad resultante del urban sprawl (Goldberg, 1999; Gregory, 2002).

Si en una primera fase este proceso se vio favorecido por la desconcentración de la industria y de la logística, el movimiento ha afectado luego con similar intensidad a un amplio abanico de actividades. Como señala Ferrão (2004, p. 518), "esta nueva realidad ha alterado la coincidencia históricamente existente entre presencia y pertenencia”, pues la vida cotidiana se organiza dentro del tejido metropolitano en torno a múltiples polos, a menudo alejados, lo que exige una creciente movilidad, con los costes económicos y ambientales consiguientes. Esto plantea especiales dificultades para lograr una gestión integrada a partir de la multiplicidad de gobiernos locales y agentes privados que en ellas operan, constituyendo uno de los retos más importantes en nuestras sociedades para avanzar hacia una más adecuada ordenación del territorio.

Por su parte, el concepto de metrópolis policéntrica o multipolar intentó describir un proceso habitual que modifica la estructura interna de estos territorios, consistente en la aparición de concentraciones secundarias de empresas y empleos en subcentros localizados, sobre todo, a lo largo de las principales vías de circulación y en los nodos intermodales de transporte - con accesibilidad incluso mayor que las áreas centrales-, llegando en ciertos casos a la construcción de lo que Garreau (1992) bautizó como ciudades de borde o edge cities. Frente al anterior proceso de suburbanización residencial, la novedad estriba ahora en la formación de áreas que integran las viviendas con áreas de actividad y empleo, lo que reduce su dependencia respecto a los espacios centrales de la aglomeración.

Según afirma Borsdorf, que identifica este fenómeno con lo que Kling, Olin y Poster (1991) o Teaford (1997) denominaron post-suburbia, "las nuevas estructuras no son zonas complementarias a la ciudad, como ocurre con las áreas suburbanas. Se distinguen de éstas por su provisión de lugares 
de abastecimiento, de trabajo, de recreación, deportivos...A la postsuburbia se trasladaron no sólo funciones centrales, como servicios de alto nivel, oficinas, parques comerciales o centros comerciales de tipo mall, entre otras (...) El resultado es una unidad espacial caracterizada por la heterogeneidad, la fragmentación de estructuras y funciones, $y$ por un patrón espacial más parecido a un patchwork que a un modelo claramente ordenado" (Borsdorf, 2005 , p. 22). Se trata, pues, de una nueva categoría espacial, poco o nada dependiente ya del centro metropolitano, al que vuelve a asociarse, de nuevo, ese viejo deseo de integrar la residencia, el trabajo, el consumo y el ocio, reduciendo así la movilidad diaria, sin considerar suficientemente la dificultad de hacer compatibles dos lógicas -las de producción de espacio residencial y de localización empresarial- que responden a criterios diferentes, ejecutadas por actores con intereses específicos, a veces contradictorios.

La ruptura de la continuidad espacial como rasgo asociado a las metrópolis difusas, o a lo que Ascher calificó como metápolis, resulta un tercer componente habitual en muchas de las interpretaciones sobre las tendencias en curso que, hasta cierto punto, convierte en obsoleto el clásico debate sobre los límites metropolitanos. Según esa perspectiva, de corte marcadamente funcionalista, es la conexión y no la contigüidad lo que permite aproximarse a unos límites metropolitanos que dejan de ser espaciales y pasan ahora a ser temporales pues, como afirma Dupuy (1998, p. 125), "el espacio pertinente ya no es el espacio continuo de los modelos geográficos clásicos, sino una tipología compleja de espacios discontinuos, desarticulados, de conexiones que generan combinaciones espacio-temporales inéditas". La metápolis desborda los anteriores conceptos de metrópolis o, incluso, de megalópolis, al tratarse de un "conjunto de espacios en los que todos o parte de sus habitantes, sus actividades económicas y sus territorios están integrados en el funcionamiento cotidiano", lo que implica "una sola cuenca de empleo, de hábitat y de actividad", si bien "los espacios que la constituyen son profundamente heterogéneos y no necesariamente contiguos" (Ascher, 1995, p. 34).

Aun sin llegar a propuestas radicales al estilo de la telépolis (Echevarría, 1994), que suponen la eliminación del espacio por el tiempo como resultado del exponencial desarrollo de las tecnologías de información y comunicación (TIC), y que difuminan la realidad metropolitana en un territorio-red compuesto de flujos materiales e inmateriales, en el que las conexiones alcanzan en ocasiones una escala mundial, también aquí la supuesta desterritorialización del fenómeno metropolitano resulta un argumento recurrente. Lo es igualmente el cambio tecnológico como principal factor de impulso, que permite-supuestamente- a los individuos y las organizaciones liberarse de las necesidades de copresencia y simultaneidad para la comunicación y el intercambio.

El conjunto de consideraciones anteriores justifican el creciente interés por analizar lo ocurrido en las periferias metropolitanas, espacios cada vez más alejados de la ciudad central, donde la velocidad absoluta y la intensidad relativa de los cambios ha sido generalmente mayor, hasta el punto de provocar una verdadera metamorfosis económico-funcional, social y morfológica, en breves periodos de tiempo. Como señala Aguilar (2006, p. 5), en muchas de ellas "las transformaciones que han tenido lugar han sido realmente dramáticas: grandes obras de infraestructura como es el caso de los aeropuertos; redes de carreteras; masivas urbanizaciones formales e informales; pérdida de grandes extensiones de suelo agrícola y otros recursos naturales, o surgimiento de complejos industriales y centros corporativos".

Pero si el dinamismo resulta, tal vez, su atributo más visible, las periferias son también territorios de transición y diversidad, en donde el impulso de la urbanización se superpone a unas sociedades, economías, culturas y paisajes rurales con desigual capacidad de resistencia. Y son, por último, territorios de oportunidad para la revitalización de las economías metropolitanas, al tiempo que de conflictos potenciales asociados a la competencia por los recursos (suelo, agua...) y a la multiplicidad de agentes, endógenos y exógenos, que operan en ellos. Pueden, por tanto, ubicarse entre los territorios inestables, "que plantean una dificultad cada vez mayor para entenderlos, aprehenderlos, construirlos intelectualmente y actuar sobre ellos a través de la planificación y el ordenamiento territorial" (Cicolella, 2006, p. 308). 
Parece indudable que buena parte de estas tendencias resultan visibles en multitud de regiones metropolitanas y la bibliografía internacional parece confirmar muchos de esos extremos. Pero una mirada más atenta a algunas diferencias también detectables entre unas y otras permite llevar a cabo una revisión crítica de algunas de esas afirmaciones, que pretende apuntar líneas de debate en futuras investigaciones:

- Las mejoras en las comunicaciones amplían de forma notable, sin duda, las posibilidades de elección para la ubicación de las firmas, pero ese potencial no basta por sí solo para transformar la realidad si no se traduce en actuaciones concretas de unos actores que lo aprovecharán o no en función de sus intereses o su capacidad para rentabilizar esas ventajas comparativas. Hace ahora un siglo, la revolución tecnológica del momento (teléfono, automóvil, tranvía, radio...) también permitió suponer que se abrían nuevas posibilidades para superar las congestionadas ciudades industriales y favorecer formas urbanas más dispersas y sostenibles, pero hoy sabemos que las décadas siguientes registraron procesos de concentración nunca conocidos hasta entonces. Por ese motivo, las actuales posibilidades desconcentradoras no deben entenderse como automáticas, sino que exigen una reflexión en cada caso sobre los actores urbanos (promotores inmobiliarios y propietarios del suelo, empresas industriales, logísticas y de servicios, gobiernos estatales, regionales y locales...) que, a partir de objetivos definidos, aplican unas u otras estrategias, lo que se traduce luego en la aprobación de determinados documentos de planeamiento, junto a fuertes inversiones en la construcción de infraestructuras, la promoción de suelo, viviendas, parques industriales, comerciales, empresariales, etc., que pueden propiciar tendencias espaciales heterogéneas.

- Los procesos en curso no parecen provocar esa dispersión aleatoria y caótica de las empresas o la población que Dear y Flusty (1998) asociaron al urbanismo posmoderno, sino más bien una aceleración de las anteriores tendencias difusoras por coronas y ejes, si bien a las conocidas geometrías de tipo axial les sustituyen otras más complejas, de carácter reticular, acordes con la propia mejora y densificación de las redes de transporte (radiales, orbitales...). En ese sentido, las dinámicas de proximidad parecen lejos de haber perdido su vigencia, tanto en términos de la elevada accesibilidad que caracteriza a los nuevos subcentros de actividad, como en la formación de clusters empresariales localizados en determinados sectores del territorio metropolitano, que a la proximidad física unen la cultural y organizativa para generar ventajas competitivas dinámicas mediante la reducción de los costes de transacción y mayores facilidades al intercambio de conocimientos tácitos, esenciales para los procesos de innovación (Dupuy y Burmeister, 2003).

- $\quad$ Parece existir, en ocasiones, cierta confusión entre los enfoques funcionales y los morfológicos, lo que conduce a perspectivas del fenómeno metropolitano que se plantean como contrapuestas cuando, en realidad, resultan complementarias. Es lo que ocurre cuando se pretende negar la posibilidad de dibujar sus contornos mediante alusiones a la existencia de territorios lejanos que mantienen vínculos funcionales diarios con la metrópoli, lo que en el plano de la información, las mercancías o el capital puede alcanzar un ámbito mundial. Las aglomeraciones metropolitanas siempre han mantenido una estrecha vinculación con territorios alejados, reflejo de su funcionalidad como centros de dirección, gestión, innovación y control de la economía-mundo capitalista, por lo que la situación actual supone una nueva fase en ese proceso; pero eso no invalida la evidencia de que la mancha urbana -que no la cultura o la economía urbanas- no se extiende de forma aleatoria o generalizada, sino que pueden establecerse unos límites -siempre convencionales y nunca bruscos- con relación a su entorno, lo que sigue haciendo útiles los intentos en esa dirección para orientar una más adecuada ordenación del territorio.

- La creciente heterogeneidad interna que caracteriza a la economía y la sociedad 
metropolitanas se acompaña por una multiplicación e intensificación de los contrastes entre las diferentes áreas que forman parte de la aglomeración, lo que hace cada vez más necesario combinar diversas escalas de análisis para lograr una descripción e interpretación adecuadas sobre el sentido e intensidad de esas desigualdades. Dentro de los mismos espacios metropolitanos coexisten empresas, actividades, grupos sociolaborales y territorios a los que puede calificarse como ganadores o perdedores, según su diversa capacidad de adaptación a las transformaciones estructurales del momento, lo que favorece la inserción y el dinamismo de unos frente a la exclusión y el declive de otros. Por esa razón, tal como propuso Veltz hace casi una década, "el territorio es cada vez menos susceptible de ser descrito en términos de macrodiferencias, como las que oponen globalmente una región a otra... En cambio, las microdesigualdades son con frecuencia muy fuertes... El territorio social y económico se vuelve a la vez más homogéneo a gran escala y más fragmentado a pequeña escala." (Veltz, 1999, p. 55).

- Más allá de su posición con respecto a la ciudad central o su accesibilidad relativa, algunas ciudades metropolitanas manifiestan un especial dinamismo y una mayor capacidad para combinar ese deseo tantas veces repetido de construir espacios competitivos y, a la vez, habitables, lo que también incluye la consolidación de entornos empresariales dinámicos e innovadores. La abundante bibliografía de la última década sobre modelos territoriales de innovación (Moulaert y Sekia, 2003) puede aportar claves para acercarse a la comprensión de esas dinámicas urbanas que no se limitan a los ámbitos metropolitanos, sino que incluyen en ocasiones a determinadas ciudades intermedias (Méndez, Michelini y Romeiro, 2006).

La posibilidad de integrar así los estudios urbanos con perspectivas más próximas a las relacionadas con el desarrollo local justifica que dediquemos a este aspecto el último apartado del artículo.
Tendencias generales vs. respuestas locales: ciudad, innovación y desarrollo

Más allá de esta interpretación general sobre las trayectorias recientes de las economías y los territorios metropolitanos, cualquier observador atento puede constatar comportamientos más o menos dinámicos e innovadores muy distintos según aglomeraciones y, en el interior de cada una de ellas, entre las diferentes ciudades que las constituyen. Sin ignorar los condicionamientos impuestos por los procesos estructurales dominantes en la actual fase de desarrollo capitalista, en los últimos años se ha prestado creciente atención a aquellos otros factores específicos que permiten a algunas ciudades aprovechar mejor sus recursos y oportunidades, frente a la escasa capacidad mostrada por el resto. Incorporar este tipo de argumentación al análisis de la actividad económica y los territorios metropolitanos supone hacer compatibles dos tipos de argumentación que aquí se entienden como complementarias, tal como refleja el diagrama de la figura 2.

Desde la clásica perspectiva de la economía urbana (Camagni, 2005), los argumentos explicativos de las ventajas competitivas de las grandes ciudades y las aglomeraciones urbanas concedieron siempre especial importancia al efecto derivado de la dimensión que, además de generar economías de escala derivadas de la indivisibilidad de algunos procesos, sería la causa fundamental de la aparición de externalidades positivas. Según esta interpretación, las mayores regiones metropolitanas del mundo desarrollado serán las que, con su acumulación de capital físico, humano, financiero $\mathrm{y}$ de conocimiento, generan el mayor volumen de economías de localización y urbanización, lo que supone un atractivo para todo tipo de actividades, en especial las de mayor contenido tecnológico y las empresas más innovadoras, lo que justifica la persistencia de fuertes tendencias polarizadoras en este tipo de funciones estratégicas. A partir de la metrópoli central, algunas de esas actividades podrán luego difundirse de forma progresiva y limitada hacia ciertas ciudades del entorno próximo $\mathrm{y}$, en su caso, otras de rango intermedio dentro del sistema urbano. Eso convierte a las aglomeraciones metropolitanas en potenciales ambientes innova- 
Figura 2. Ventajas competitivas y procesos de desarrollo local en espacios metropolitanos.

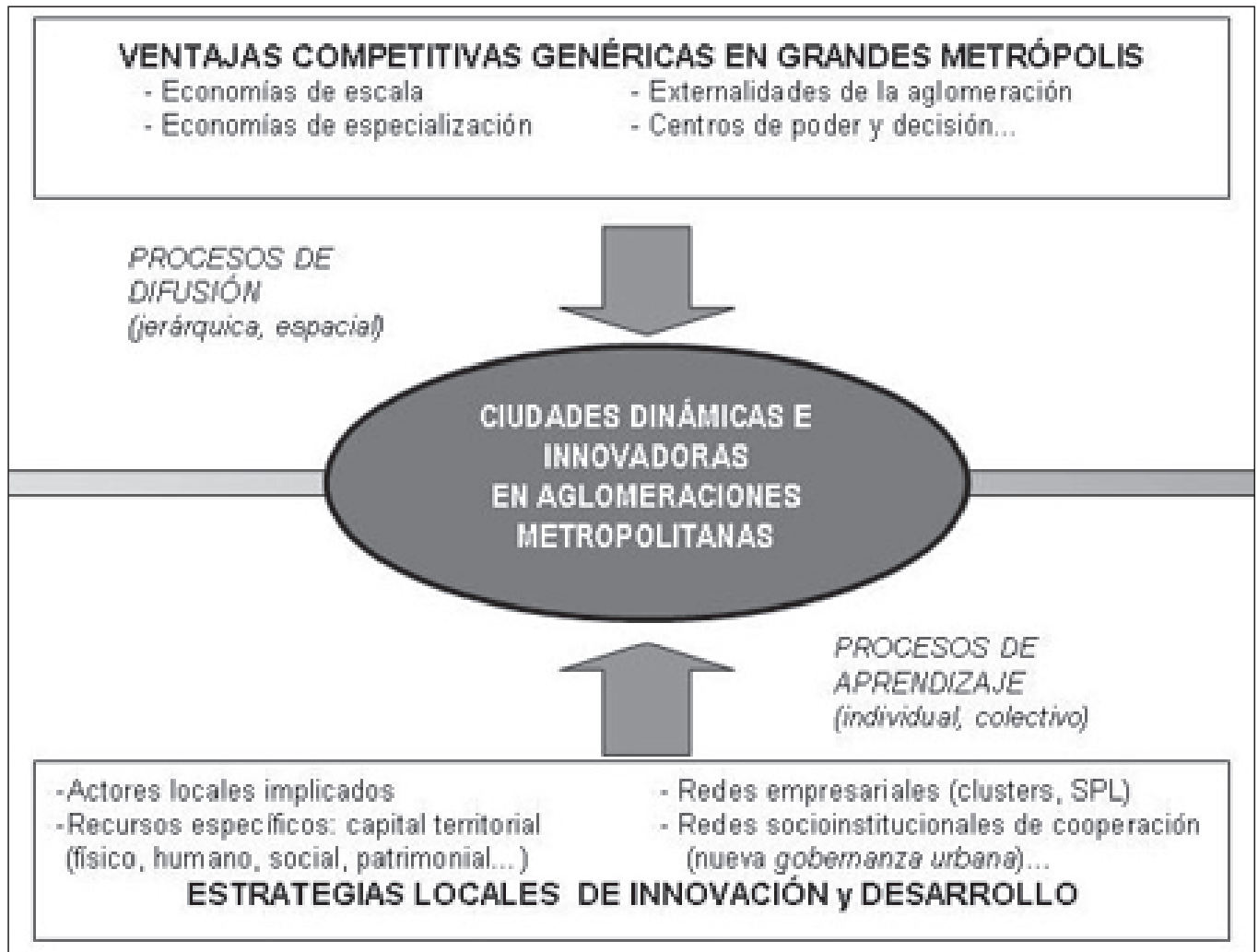

Fuente. Elaboración propia.

dores, pese a que la activación de ese potencial resulta claramente distinta según los casos.

No obstante, tanto los estudios sobre la diversa empresarialidad de los territorios como, sobre todo, toda la literatura que en la década pasada se vinculó a la idea de los milieux o entornos innovadores (Maillat y Kébir, 1998; Crevoisier y Camagni, 2000) y en la actual a las denominadas ciudades inteligentes y ciudades creativas (Cooke y Morgan, 1998; Scott, 2006), las razones del dinamismo y la capacidad innovadora -tanto económica como socialtienen, ante todo, una raíz endógena, en la que factores económicos y socioinstitucionales se complementan.

En esa interpretación, un primer factor a considerar son los recursos locales puestos a disposición de las empresas. Parecen de especial importancia aquellos que resultan específicos del ámbito que los ha producido, constituyendo lo que puede considerarse como su capital territorial. Frente a su concepción como simple acumulación de factores productivos o yacimiento de externalidades, la ciudad se entiende así como construcción social específica en donde, a partir de unas dotaciones iniciales variables, algunas sociedades son capaces de generar un escenario favorable a la innovación mediante la utilización de recursos específicos que hacen posible la creación de unas condiciones sólo entendibles en relación con ese ámbito. Entre los principales recursos específicos, que deben convertirse en activos movilizados por la sociedad local, se encuentran el capital físico o infraestructural (desde comunicaciones a servicios avanzados, centros educativos de calidad, instituciones de producción o transferencia de conocimiento...), el capital humano (población con cierto nivel formativo, saber hacer local, iniciativa empresarial...), el capital productivo (estructura empresarial heredada, cultura empresarial...), el financiero o el patrimonial. 
Pero, como ha puesto de manifiesto una abundante literatura en años recientes, se considera recurso de especial importancia el marco institucional, directamente ligado a la noción de capital social. Según la clásica definición de North (1990, p. 3), las instituciones se entienden como "las reglas del juego de una sociedad o, de modo más formalizado, los límites definidos para modelar la interacción humana", que tienden a facilitar e, incluso, estimular, ciertos comportamientos colectivos, desanimando otros. En ese sentido, las ciudades con mayor densidad institucional presentarán ciertos valores culturales y reglas implícitas de comportamiento colectivo, que en los casos más evolucionados se trasladan a normas y organizaciones formalizadas y estables, favorables al desarrollo de iniciativas innovadoras y a procesos de aprendizaje colectivo derivados de la participación y la cooperación. Eso propicia también un mayor anclaje territorial de las empresas, al tratarse de recursos difíciles de encontrar en otras localizaciones, así como una mayor diferenciación de sus productos y servicios.

La producción de esos recursos específicos requiere la presencia de actores locales, con voluntad y capacidad de dinamizar la sociedad urbana y promover iniciativas, tanto de movilización y captación de recursos financieros, difusión de información y conocimiento, o inserción exterior, perspectiva especialmente destacada por las llamadas teorías de la acción (Jambes, 2001). Las metáforas relativas a la existencia de ciudades con proyecto, ciudades que aprenden, etc., aluden a esa capacidad potencial de los actores locales -públicos, privados o, a ser posible, de ambos tipos- para diagnosticar las debilidades y las amenazas potenciales, definir objetivos y aportar recursos y capacidades para alcanzarlos.

En consecuencia, identificar qué actores operan en cada caso y su escala de actuación, conocer y comprender sus características, intereses y valores, los mecanismos que guían el proceso de decisión, las estrategias que aplican para alcanzar sus objetivos, o sus posibles interacciones (colaboración, competencia, conflicto) son aspectos relevantes en la interpretación del desigual desarrollo que muestran diferentes metrópolis.
Un tercer factor de impulso en la construcción de ciudades innovadoras es la presencia de una organización de sus diferentes actividades económicas de tipo sistémico. La densidad y estabilidad de las relaciones de interdependencia entre empresas urbanas, ya sean de carácter mercantil (compraventa entre proveedores y clientes, subcontratación...), o bien al margen del mercado (acuerdos de colaboración formales o informales, intercambio de información...), constituyen la base de los sistemas productivos locales, que tanto interés han despertado en las dos últimas décadas.

La simple concentración geográfica asociada a la presencia de clusters localizados ya genera cierto volumen de economías externas, que pueden ser aprovechadas de forma pasiva por las empresas allí localizadas; no obstante, éstas "son ventajas estáticas que no bastan cuando las empresas de la aglomeración tienen que enfrentarse con situaciones de cambio que inciden negativamente en su capacidad de competir" (Dini y Stumpo, 2004, p. 13). Por el contrario, otras dinámicas asociadas a la proximidad física, cultural y organizativa entre las empresas locales, que además de reducir los costes de transacción favorecen las relaciones de confianza entre individuos y, de ese modo, la transmisión de conocimientos tácitos y no formalizados, son las que propician la colaboración en acciones conjuntas y, a través de ellas, el aprendizaje colectivo, convirtiéndose así en fuentes de ventajas competitivas dinámicas que permiten hablar de una verdadera eficiencia colectiva (Di Tommaso y Rabellotti, 1999).

Un último factor a destacar en esta nueva manera de interpretar el dinamismo urbano y su capacidad de inducir el desarrollo territorial señala que para comprender los procesos de innovación en el seno de las empresas y de los sistemas productivos urbanos hay que trascender el ámbito estrictamente económico para fijarse en la posible existencia de redes sociales e institucionales de apoyo. Así pues, para intensificar los procesos de aprendizaje colectivo es necesario que los múltiples integrantes de los sistemas locales de innovación -con especial protagonismo de los gobiernos locales, las organizaciones empresariales y sindicales- establezcan ciertas relaciones de cooperación a 
Figura 3. Influencia de las instituciones y el capital social sobre los procesos de innovación urbana.

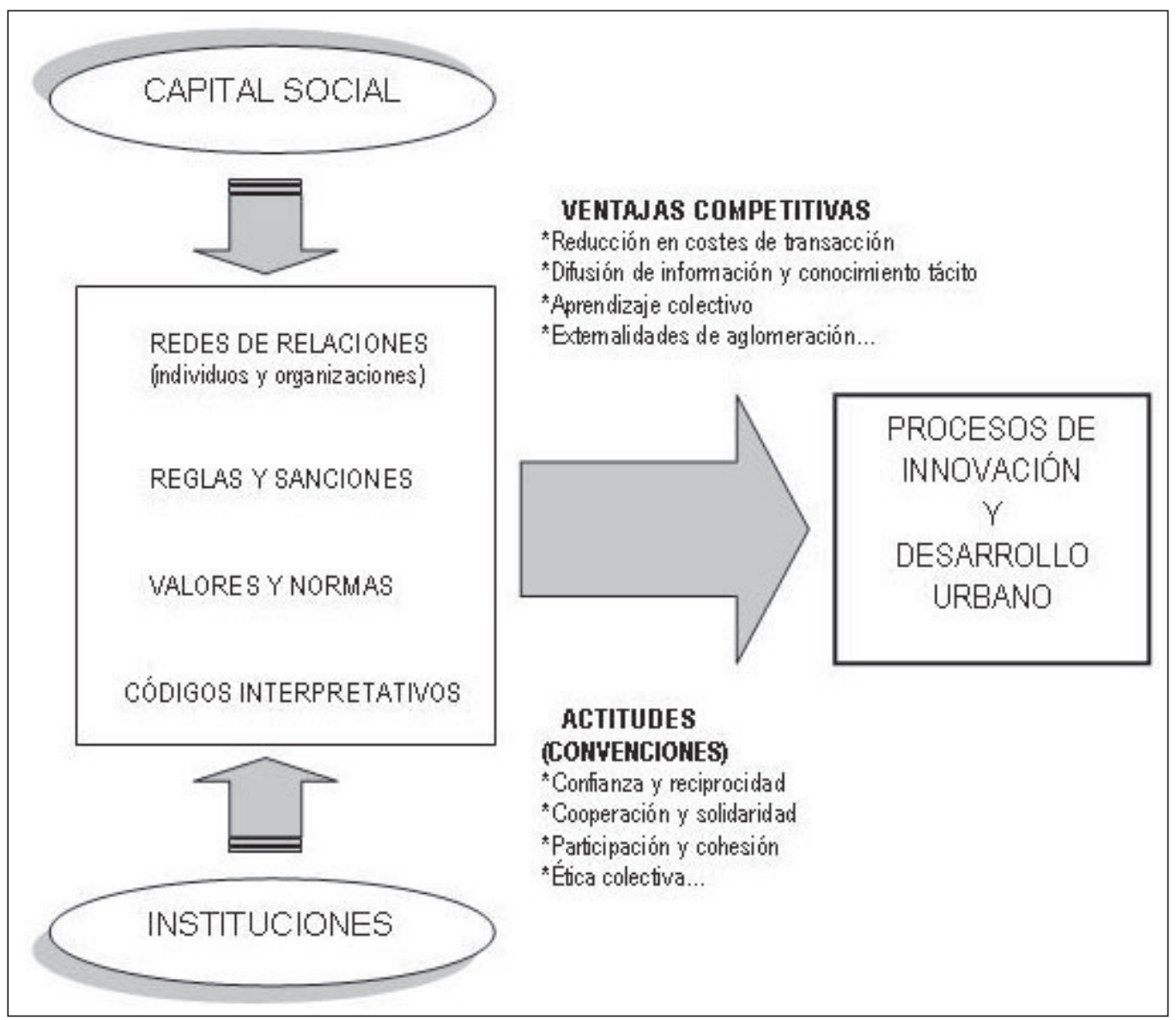

Fuente. Elaboración propia.

medio plazo, lo que exige la creación de un clima de confianza, generalmente difícil de alcanzar. Por el contrario, la falta de redes locales de cooperación puede entenderse como un obstáculo en el camino de la innovación y algo similar puede afirmarse de la escasa implicación de los poderes locales en la generación de proyectos comunes.

Particular atención se concede en los últimos años a la capacidad de concertación entre los diferentes niveles de gobierno con incidencia en la ciudad y de éstos con los actores privados, orientada a promover una nueva gobernanza en la que el poder "no está asignado ni adquirido de forma definitiva, sino, sobre todo, es ejercido y negociado a través de relaciones en las que se expresa un reparto de responsabilidades" (Barthe, 1998, p. 6). Al igual que ocurre con las redes empresa- riales, en bastantes ocasiones las relaciones que se observan tienen un carácter circunstancial y una escasa formalización pero, incluso en esos casos, la presencia de lo que Granovetter (1985) calificó como la fuerza de los vínculos débiles permite la aparición de formas de solidaridad conmutativa que relaciona a individuos y organizaciones pertenecientes, a su vez, a múltiples redes, haciendo posible su participación conjunta en proyectos que se juzgan de interés colectivo. Según Ascher, esta gobernanza urbana define un modo de regulación que califica de societario, "en la medida en que actores con lógicas diferentes e intereses posiblemente divergentes o incluso contrapuestos sobre una serie de puntos, intentan o se ven obligados a una gestión conjunta, a negociar compromisos duraderos y crear instituciones colectivas", al 
tiempo que reflexivo, "adaptado a una sociedad abierta, diversificada, móvil e inestable" (Ascher, 2004, pp. 51-52).

Esta referencia a las redes sociales de apoyo a la innovación -identificadas por Camagni (2003) como capital relacional- que pueden propiciar también economías urbanas más competitivas, remiten de nuevo a la cuestión de las instituciones y el capital social, ya mencionada con anterioridad.

Mientras la visión defendida por Putnam (1993) sobre el capital social vincula la existencia de un marco institucional dominado por valores, normas y códigos favorables al desarrollo local a las herencias culturales acumuladas en sociedades específicas, que maduran y predisponen al establecimiento de relaciones de confianza, reciprocidad o participación, indispensables para la puesta en marcha de proyectos de colaboración, "hay también formas de capital social que derivan de una inversión específica en la creación de estructuras capaces de generarlo" (Bagnasco, 2000, p. 84). Éste es el caso de las organizaciones públicas o privadas creadas con objeto de reforzar la capacidad local de innovación, así como de las redes destinadas al fomento de la cooperación.

En consecuencia, la formalización de organizaciones y redes dotadas de reglas orientadas en esa dirección es la que permite activar un recurso específico como puede ser un tejido institucional proclive a la innovación a partir de acciones concretas de los actores locales, lo que también ayuda a comprender las grandes diferencias existentes a este respecto en sociedades urbanas con un bagaje cultural muy similar. El esquema de la figura 3 intenta reflejar esta interpretación sobre la doble vía de acceso a la innovación urbana, bien a partir de lo que Pizzorno (2003) denomina capital social de solidaridad, asociado a la presencia de cierta cohesión social derivada de herencias culturales compartidas, o bien a partir de un capital social de reciprocidad, construido de forma intencional por actores locales unidos por lazos a menudo débiles, pero con intereses comunes y capacidad para traducirlos en acciones efectivas.

\section{Algunas consideraciones finales}

Todo intento, como el aquí realizado, de llevar a cabo una revisión crítica sobre las interrelaciones actuales entre actividades económicas y espacios metropolitanos exige poner el énfasis en la búsqueda de una propuesta interpretativa capaz de generar unos esquemas básicos que permitan orientar un recorrido por la gran cantidad y variedad de información, conceptos y teorizaciones disponibles en la actualidad, cuestionando algunos caminos y proponiendo algunas vías a explorar. Pero no parece oportuno finalizar sin una mención, siquiera telegráfica, a dos aspectos que parecen exigir una cierta reflexión en clave de futuro.

Por un lado, parece evidente que "la creciente integración económica continúa siendo un proceso contradictorio e incompleto, generador de divisiones, desigualdades y tensiones" (Hudson, 2003, p. 55) y, en consecuencia, las formas territoriales específicas generadas por el funcionamiento del sistema están lejos de desaparecer, aunque se transformen sus manifestaciones externas para responder a la lógica imperante en esta nueva fase de desarrollo capitalista. Como resultado de una división espacial del trabajo que acentúa sus rasgos y multiplica sus escalas, se refuerza la especialización funcional de las metrópolis, con una dinamización selectiva de los enclaves que albergan aquellos sectores y empresas más competitivos, frente al declive de otras actividades y espacios. En ese contexto, es indudable que el peso relativo de las funciones de comando tiende a aumentar en ellas de forma generalizada, provocando una "centralización del control" en los principales nodos que articulan las redes de negocios globalizadas (Sassen, 2003), lo que justifica la creciente atención que se les presta. Pero parece, en cambio, más discutible el relativo olvido de otra serie de actividades -tanto productivas como de servicios- que continúan cimentando una base económica diversificada en muchas de nuestras metrópolis, cuya capacidad o incapacidad para construir entornos locales innovadores seguirá siendo esencial para su futuro económico.

Por otra parte, en una parte de la literatura reciente sobre la transformación que experimentan estos espacios, se corre el riesgo de confundir lo complejo con lo caótico, abandonando por inútiles los esfuerzos por discernir las nuevas 
formas metropolitanas asociadas a los procesos de índole económica, tecnológica o sociocultural. La proliferación de neologismos que reiteran -bajo muy diversas denominaciones- la generalización de aglomeraciones de baja densidad, discontinuas y aparentemente informes, resulta una descripción, a veces brillante pero impresionista, de una tendencia que debe someterse a revisión, cuando se constata que tanto los diferentes sectores de actividad, empresas, funciones o tipos de empleo se reestructuran con rapidez, pero manteniendo una lógica espacial bastante estricta en sus comportamientos. Sin llegar a analogías a menudo excesivas, como las de tipo fractal, se necesitan aún cartografías más precisas, unidades espaciales más adecuadas y una revisión de las clasificaciones heredadas, para perfilar mejor la nueva geografía económica metropolitana, distinguiendo las tendencias comunes de las singularidades propias de cada lugar.

Hace ahora dos décadas, en la presentación de un documento que resumía los primeros estudios promovidos por la administración pública en Francia sobre mutaciones económicas y urbanización, los redactores del mismo escribían: "el periodo actual está marcado por mutaciones espaciales y urbanas profundas, que están ligadas, al menos en parte, a las transformaciones económicas en curso. El conocimiento de la articulación entre esos dos campos no ha sido objeto de inversiones recientes en investigación a la altura del problema. Los responsables políticos, económicos y los técnicos en ordenamiento perciben - cada uno a partir de su ámbito de intervención- aspectos parciales de esas articulaciones, pero tienen dificultades para interpretarlas y, sobre todo, para medir las consecuencias derivadas" (VVAA, 1987, p. 15). Es innegable que mucho se ha escrito al respecto desde entonces, pero el propio dinamismo del sistema genera cambios acelerados y nuevas contradicciones que justifican continuar la reflexión sobre su significado y, más aún, aportar evidencias empíricas sustantivas que permitan elaborar propuestas de intervención más adecuadas.

\section{Referencias bibliográficas}

Aguilar, A. G. (2002). Las mega-ciudades y las periferias expandidas. Ampliando el concepto en Ciudad de México. EURE, 28, 85, 121150.

(Coord.) (2006). Las grandes aglomeraciones y su periferia regional. Experiencias en Latinoamérica y España. México: Miguel Ángel Porrúa-UNAM

Améndola, G. (2000). La ciudad postmoderna. Madrid: Celeste Ediciones.

Antonelli, C. y Ferrâo, J. (Coord.) (2001). Comunicaçâo, conhecimento colectivo e inovaçâo. As vantagens da aglomeraçâo geográfica. Lisboa: Instituto de Ciencias Sociais.

Ascher, F. (1995). Metapolis ou l'avenir des villes. París: Odile Jacob.

(2004). Los nuevos principios del urbanismo. Madrid: Alianza Editorial.

Bagnasco, A. (2000). Nacimiento y transformación de los distritos industriales. Un examen de la investigación en Italia con observaciones de método para la teoría del desarrollo. En M. Carmagnani \& G. Gordillo (Coords.). Desarrollo social y cambios productivos en el mundo rural europeo contemporáneo (pp. 59-91). México: Fondo de Cultura Económica.

Barthe, L. (1998). La construction politique du territoire dans les strategies de développement local. Geodoc, 46, 5-9.

Borsdorf, A. (2005). La transformación urbana-rural en Europa. ¿Hacia una unificación espacial en post-suburbia? En C. de Mattos et al. (Eds.) Gobernanza, competitividad y redes. La gestión de las ciudades en el siglo XXI (pp. 21-30). Santiago: Pontificia Universidad Católica de Chile.

Camagni, R. (2003). Incertidumbre, capital social y desarrollo local: enseñanzas para una gobernabilidad sostenible del territorio. Investigaciones Regionales, 2, 31-58.

(2005). Economía urbana. Barcelona: Antoni Bosch Editor.

Caravaca, I. \& Méndez, R. (2003). Trayectorias industriales metropolitanas: nuevos procesos, nuevos contrastes. EURE, 29, 87, 37-50.

Caravaca, I. (2006). La nueva industria urbana y metropolitana: procesos, estrategias y resultados. En R. Méndez \& H. Pascual (Eds.) Industria y ciudad en España: nuevas realidades, nuevos retos (pp. 29-50). Madrid: Thomson Civitas. 
Castells, M. (1997). La era de la información. Economia, sociedad y cultura. Volumen I: La sociedad red. Madrid: Alianza Editorial.

Ciccolella, P. \& Lucioni, N. (2005). La ciudad corporativa. Nueva arquitectura empresarial, redefinición de la centralidad y surgimiento de una red de distritos de comando en la Región Metropolitana de Buenos Aires. En C. de Mattos et al. (Eds.). Gobernanza, competitividady redes. La gestión de las ciudades en el siglo XXI (pp. 185-209). Santiago: Pontificia Univ. Católica de Chile.

Ciccolella, P. (2006). Metrópolis latinoamericanas: territorios subregulados, ¿espacios del capital? En A.G. Aguilar (Coord.), Las grandes aglomeraciones y su periferia regional. Experiencias en Latinoamérica y España (pp. 305-324). México: Miguel Ángel Porrúa-Cámara de DiputadosConacyt-UNAM.

Cooke, P. N. \& Morgan, K. (1998). The associational economy: firms, regions and innovation. Oxford: Oxford University Press.

Crevoisier, O. \& Camagni, R. (Eds.) (2000). Les milieux urbains: innovation, systèmes de production et ancrage. Neuchâtel: EDES.

Cuadrado Roura, J. R. \& Fernández Güell, J. M. (2005). Las áreas metropolitanas frente al desafío de la competitividad. En E. Rojas, J. R. Cuadrado \& J. M. Fernández (Eds.), Gobernar las metrópolis (pp. 63-125). Washington: Banco Interamericano de Desarrollo.

Daniels, P. W. \& Bryson, J. R. (2002). Manufacturing services and servicing manufacturing: knowledge-based cities and changing forms of production. Urban Studies, 39, 5-6, 977-991.

De Mattos, C. (1998). Reestructuración, crecimiento y expansión metropolitana en las economías emergentes latinoamericanas. En S. Gorenstein y R. Bustos (Comps), Ciudades y regiones frente al avance de la globalización (pp. 13-38). Bahía Blanca: Universidad Nacional del Sur.

De Mattos, C.; Figueroa, O.; Giménez, R.; Orellana, A. \& Yáñez, G. (Eds.) (2005). Gobernanza, competitividad y redes. La gestión de las ciudades en el siglo XXI. Santiago: Pontificia Universidad Católica de Chile.

De Santiago, E. (2006). Nuevas formas y procesos espaciales en la región urbana de Madrid: las lógicas del espacio en la construcción de la ciudad única. Te- sis doctoral no publicada, ETS Arquitectura, Madrid, España.

Dear, M. \& Flusty, S. (1998). Postmodern urbanism. Annals of the Association of American Geographers, 88, 1, 50-72.

Dematteis, G. (1998). Suburbanización y periurbanización. Ciudades anglosajonas y ciudades latinas. En F. Monclús (Ed.), La ciudad dispersa. Barcelona: Centre de Cultura Contemporània.

Di Tommaso, M. \& Rabellotti, R. (Comps.) (1999). Efficienza collettiva e sistemi d'imprese. Bolonia: Il Mulino.

Dini, M. \& Stumpo, G. (Coords.) (2004). Pequeñas y medianas empresas y eficiencia colectiva. Estudios de caso en América Latina. México: Siglo XXI-CEPAL.

Dupuy, C. \& Burmeister, A. (2003). Entreprises et territoires. Les nouveaux enjeux de la proximité. París: La Documentation Française.

Dupuy, G. (1998). El urbanismo de las redes. Teorías y métodos. Barcelona: Oikos-Tau.

Echevarría, J. (1994). Telépolis. Barcelona: Destino.

Ferrão, J. (2004). Las regiones metropolitanas como comunidades imaginadas: vivencias, discursos y acción. Ciudad y Territorio Estudios Territoriales, 36, 141-142, 517-522.

Florida, R. (2002). The rise of the creative class. Nueva York: Basic Books.

Fujita, M.; Krugman, P. \& Venables, A. J. (2000). Economía espacial. Las ciudades, las regiones y el comercio internacional. Barcelona: Ariel.

Gaja, F. (2004). Evidencias e hipótesis sobre la ciudad informacional. Ciudady Territorio Estudios Territoriales, 36, 141-142, 507-522.

García Palomares, J.C. (2007). Movilidad laboral en la Comunidad de Madrid. Tesis doctoral no publicada, Universidad Complutense, Madrid, España.

Garreau, J. (1992). Edge city: life in the new frontier. Nueva York: Doubleday.

Gilly, J. P. \& Torre, A. (Dirs.) (2000). Dynamiques de proximité. París: L'Harmattan.

Goldberg, D. (1999). Covering urban sprawl. Rethinking the American dream. RTNDF Environmental Journalism Center.

Granovetter, M. (1985). Economic action and social structure: the problem of embeddedness. American Journal of Sociology, 91, 3, 481-510. 
Gregory, D. (Ed.) (2002). Urban sprawl. Causes, consequences \& policy responses. Washington: Urban Institute Press.

Harvey, D. (1990). The condition of postmodernity. Oxford UK: Blackwell.

Hudson, R. (2003). European integration and new forms of uneven development. But not the end of territorially distinctive capitalism in Europe. European Urban and Regional Studies, 10, 1, 49-67.

Jambes, J. P. (2001). Territoires apprenants. Esquisses pour le développement local du XXIe siécle. París: L'Harmattan.

Kling, R.; Olin, S. \& Poster, M. (Eds.) (1991). Postsuburbian California: the transformation of postwar Orange County, California. Irvine: University of California Press.

Lauria, M. (Ed.) (1997). Reconstructing urban regiome theory. Beverly Hills: Sage Publications.

Maillat, D. \& Kebir, L. (1998). Learning region et systèmes territoriaux de production. Working Paper IRER, 9802a, Université de Neuchâtel, Neuchâtel, Suiza.

Méndez, R. (2006). La mutation des marchés métropolitains du travail: realités et mythes dans la région de Madrid. En R. Méndez (Ed.), Géographie de l'Espagne (pp.137-162). París: L'Harmattan.

Méndez, R.; Michelini, J. J. \& Romeiro, P. (2006). Redes socioinstitucionales e innovación para el desarrollo de ciudades intermedias. Ciudad y Territorio Estudios Territoriales, 38, 148, 377 395.

Mollenkopft, H. \& Castells, M. (1992). The dual city. Nueva Cork: Rusell Sage Fondation.

Moulaert, F. \& Sekia, F. (2003). Territorial innovation models: a critical survey. Regional Studies, 37, 3, 289-302.

Nel.lo, O. (1998). Los confines de la ciudad sin confines. Estructura urbana y límites admi- nistrativos en la ciudad difusa. En J. Monclús (Ed.). La ciudad dispersa. Suburbanización y nuevas periferias (pp. 35-57). Barcelona: Centre de Cultura Contemporània. North, D. (1990). Institutions, institucional change and economic performance. Cambridge: Cambridge University Press.

OCDE (2006a). Competitive cities in the global economy. París: OECD.

(2006b). Innovation and knowledge-intensive service activities (KIS A). París: OECD.

Pizzorno, A. (2003). Por qué pagamos la nafta. Por una teoría del capital social. En C. Trigilia et al, El capital social. Instrucciones de uso (pp. 19-52). Buenos Aires: Fondo de Cultura Económica.

Pradilla, E. \& Márquez, L. (2004). Estancamiento económico, desindustrialización y terciarización informal en la ciudad de México (19802003) y potencial de cambio. Investigación y Diseño, 1, 143-161.

Putnam, R. D. (1993). Making democracy work. Princeton: Princeton University Press.

Ramirez, B. (2006). Del funcionalismo industrial al de servicios: ¿La nueva utopía de la metrópoli postindustrial del valle de México? EURE, $32,95,61-74$.

Sassen, S. (2003). Localizando ciudades en circuitos globales. EURE, 29, 88, 5-27.

Scott, A. J. (2006). Creative cities: conceptual issues and policy questions. Journal of Urban Affairs, 28, 1, 1-17.

Soja, E. (2000). Postmetropolis. Oxford: Blackwell.

Teaford, J. C. (1997). Post-suburbia: government and politics in the edge cities. Baltimore: Johns Hopkins University Press.

Veltz, P. (1999). Mundialización, ciudades y territorio. Barcelona: Ariel.

VV.AA. (1987). Mutations économiques et urbanisation. París: La Documentation Française. 